\title{
A Molecular-Rotor Device for Nonvolatile High-Density Memory Applications
}

\author{
Mei Xue, Student Member, IEEE, Sanaz Kabehie, Adam Z. Stieg, Ekaterina Tkatchouk, Diego Benitez, \\ William A. Goddard, Jeffrey I. Zink, and Kang L. Wang, Fellow, IEEE
}

\begin{abstract}
A novel memory device based on an electrically driven molecular rotor was fabricated and demonstrated to have bistable switching effects. The device showed an on/off ratio of approximately $10^{4}$, a read window of about $2.5 \mathrm{~V}$, and retention performance of greater than $10^{4} \mathrm{~s}$. The analysis of the device $I-V$ characteristics suggests the source of the observed switching effects to be the redox-induced ligand rotation around the copper metal center, which is consistent with the observed temperature dependence of the switching behavior. This organic monolayer device holds a potential for nonvolatile high-density memory applications due to its scalability and reduced cost.
\end{abstract}

Index Terms-Molecular rotor, monolayer, nonvolatile memory, redox-induced rotation, resistive switching, tunneling transport.

\section{INTRODUCTION}

A S PHYSICAL fabrication limits are approached, miniaturization, a driving force of the semiconductor industry, faces challenges in further scaling basic microelectronic circuit components such as memory and switches. Nanoscale electronics based on functional molecular units acting as a state variable provides an attractive alternative to overcome the limits of conventional MOSFET technology due to their potential scalability, low-cost, highly integrated characteristics, and the capability to exploit self-assembly processes [1]-[3]. Triggered by external stimuli, such as electrical signal or chemical reagents, these devices have shown various functions, including rectifiers [1], memories [4]-[6], and single settable molecular switches that can be electronically configured for logic gates. Although these molecular devices offer excellent scaling potential, their applications are limited due to low yield, low on/off ratio, and slow programming speed.

In this letter, we demonstrate an electrically driven sandwichtype monolayer molecular-rotor device showing a bistable resistive switching effect for the first time. The rotation speed of this type of molecular rotor has been reported to be in the picosecond timescale, which provides a potential of high

Manuscript received May 17, 2010; accepted May 26, 2010. Date of publication July 19, 2010; date of current version August 25, 2010. This work was supported by the FCRP-FENA Center. The review of this letter was arranged by Editor T. Wang.

M. Xue, S. Kabehie, A. Z. Stieg, J. I. Zink, and K. L. Wang are with the California NanoSystems Institute, University of California, Los Angeles, CA 90095-7277 USA (e-mail: mxue@ee.ucla.edu; sanaz@ chem.ucla.edu; stieg@cnsi.ucla.edu; zink@chem.ucla.edu; wang@ee.ucla.edu).

E. Tkatchouk, D. Benitez, and W. A. Goddard are with the Materials and Process Simulation Center, California Institute of Technology, Pasadena, CA 91125 USA (e-mail: ekat@caltech.edu; diego@caltech.edu; wag@wag. caltech.edu).

Color versions of one or more of the figures in this letter are available online at http://ieeexplore.ieee.org.

Digital Object Identifier 10.1109/LED.2010.2052018

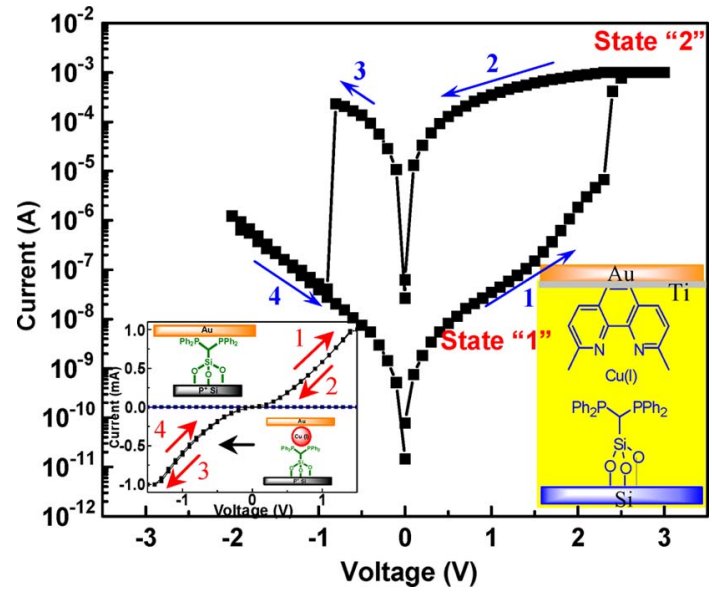

Fig. 1. $I-V$ characteristics of the (complete) monolayer device with the bisP stator. The inset shows the $I-V$ characteristics of the molecular layer devices composed of solely grafted stators or Cu-ligated stator subunits only and the $\mathrm{Cu}(\mathrm{I})$ structure.

switching speed [7], [11]. This switching effect holds a great potential for high-density memory applications.

\section{Device Fabrication}

The functional unit of this molecular device is composed of heteroleptic copper compounds, which in turn consist of a stator unit (a bidentate ligand), an axle unit $[\mathrm{Cu}(\mathrm{I})]$, and a rotator unit (2,9-dimethyl-1,10-phenanthroline) as shown in the inset of Fig. 1. This copper compound exhibits two discrete redoxdependent conformational states, $\mathrm{Cu}(\mathrm{I})$ and $\mathrm{Cu}(\mathrm{II})$, resulting in different transport paths for electrons. The $\mathrm{Cu}(\mathrm{I})$ form has tetrahedral geometry, while the $\mathrm{Cu}(\mathrm{II})$ form is square planar. The compounds undergo a one-electron redox-induced rotational conformational change depending on the oxidation state of the copper metal. Interconversion between these two states provides the basis for a controlled bistable nanoswitch.

Fabrication of the molecular-rotor device employed a surface outward sequential synthesis on $10^{19} \mathrm{~cm}^{-3}$ doped $\mathrm{p}+$ Si substrate based on self-assembly in order to ensure the formation of immobilized heteroleptic copper compounds capable of $90^{\circ}$ relative rotational motion of the ligands [7]. Preparation of the heteroleptic copper system was carried out through covalent grafting of a stator monolayer ([bis(diphenylphosphino)methyl]triethoxysilane onto the hydroxylated surface of a $\mathrm{P}^{+}-\mathrm{Si}$ substrate using silanol bonds. Propyltriethoxysilane was then added to fill in any gap between immobilized stators and to minimize background current. The stators were then used to chelate a copper metal axle that subsequently bonds to the rotator subunit. Atomic-force-microscopy 
imaging demonstrated the successful preparation of a molecular monolayer. The device was completed by deposition of a Ti/gold film through a shadow mask on top of the molecular layer to form the top electrode. The active area of the device was about $3 \times 10^{-4} \mathrm{~cm}^{2}$.

\section{RESUlTS AND DISCUSSION}

The $I-V$ characteristics of the molecular-rotor device with a semilogarithmic current scale are shown in Fig. 1. In this case, the silicon substrate represents the system ground, and the dc bias is applied from the top gold electrode. The bias sweep direction followed the arrows numerically. State " 1 " represents the high-resistance state (HRS/off) and State "2" is the lowresistance state (LRS/on), where the turning voltage between State 1 and State 2 is determined by the redox energy of the copper compound. Beginning with the device residing in the OFF state, application of a positive bias of $>2.2 \mathrm{~V}$ switches the device to an ON state and demonstrates a drastic increase in conductivity. Switching to the OFF state is achieved by applying a negative bias of $-1 \mathrm{~V}$, producing a corresponding abrupt decrease in conductivity. The device remains in the OFF state until a positive turn-on voltage is applied. The on/off current ratio is about four orders of magnitude, and the read voltage window is about $2.5 \mathrm{~V}$. (If one polarity supply is used, the window would be $2.5 \mathrm{~V}$. However, if the threshold voltage can be shifted, then a $3.5 \mathrm{~V}$ window can be achieved.)

To further explore the correlation of the observed switching effect with redox-induced conformational switching, $I-V$ characteristics of devices whose molecular layer consisted of solely grafted stators or $\mathrm{Cu}$-ligated stators were investigated, as shown in the inset of Fig. 1. Both devices did not show any hysteretic switching effects. Since these devices lack the capability for rotational motion because of a missing ligated rigid rotator, this phenomenon indicates that the observed switching effects in full molecular devices do not stem from either the subunit ligands themselves or the interfaces between the ligands and the metal electrodes. Therefore, the lack of the observed switching effects in these cases implies the source of the observed switching effects in full devices to be a result of conformational effects. The retention times of both the LRS and HRS were measured to be on the order of $10^{4} \mathrm{~s}$ with very little degradation, as shown in Fig. 2, a result indicative of relative stability for both states. The retention times should be better with proper packaging to isolate the device from the atmosphere. Moreover, for a given device, the $I-V$ characteristics are stable and reproducible with consecutive positive-and negative-bias sweeps.

To investigate the operation mechanism of the device, the experimental data of State 1 undergoing a transition from HRS to LRS state are analyzed, as seen in Fig. 3. Beginning in State 1 (HRS) of the $\mathrm{Cu}$ (II) complex under a small positive bias, tunneling transport dominates the measured current. This can be explained as follows: An experimental conductance-voltage plot (Fig. 3(a) and inset) demonstrated a roughly parabolic dependence at low voltages $(\leq \pm 0.3 \mathrm{~V})$ as compared with an exponential relationship at higher voltages $(>-1 \mathrm{~V})$. This agreement between the measured results and the theoretical fitting implies that the State 1 current can be attributed to tunneling current between the two electrodes based on Rowell's second criterion ([8]). (The weak temperature dependence of the experimental results, shown in Fig. 4, further supports the

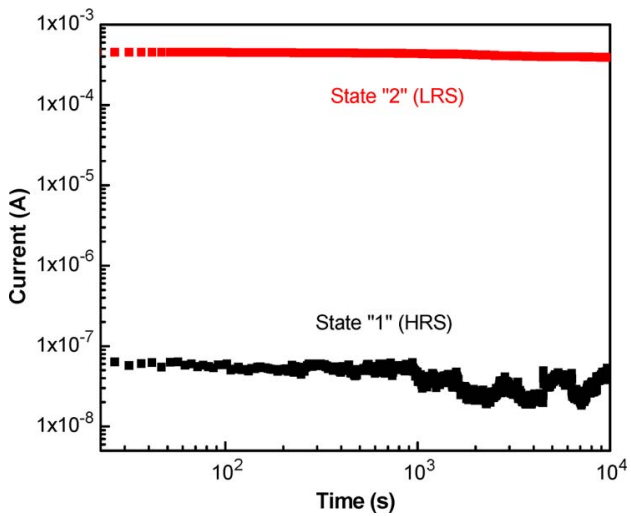

Fig. 2. Retention-time measurement of the ON and OFF states under an applied bias of $1 \mathrm{~V}$ at room temperature.

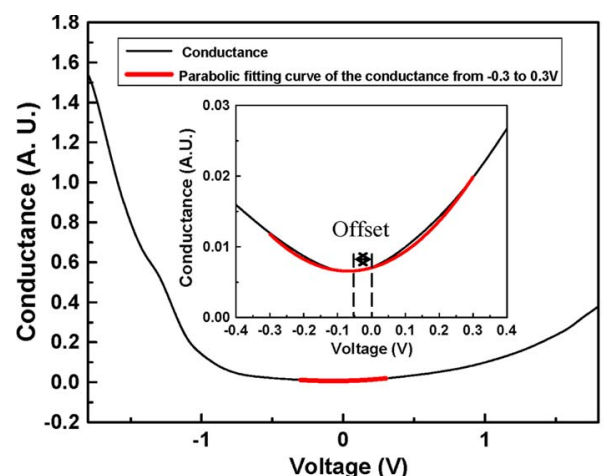

(a)

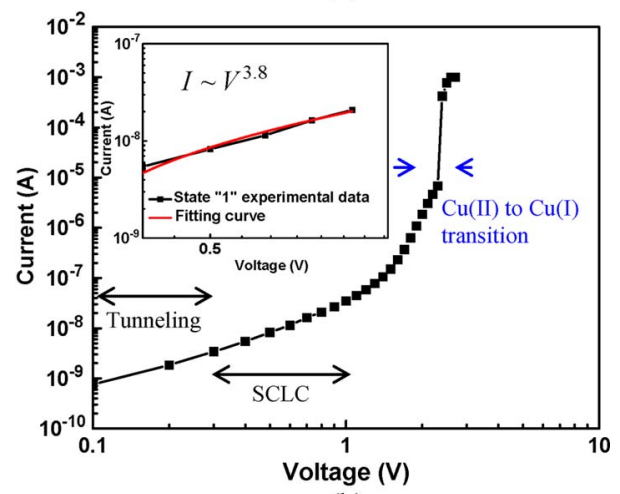

(b)

Fig. 3. (a) Parabolic fitting of the conductance-voltage plot at low voltages $(\leq 0.3 \mathrm{~V})$ shown as the inset. (b) State 1 current plotted in the log scale. The inset shows the SCLC in the range of $0.3-1.2 \mathrm{~V}$.

tunneling-transport mechanism.) The offset of this parabola of about $-0.05 \mathrm{~V}$ from $V=0$ is the result of asymmetry in the potential-barrier shape. In the range between 0.3 and $1.2 \mathrm{~V}$, however, the measured current follows a power law of $I-V^{3.8}$ rather than an exponential relationship, as shown in the inset of Fig. 3(b). This $I-V$ relationship is indicative of space-charge-limited current (SCLC) transport. According to the SCLC model, the $I-V$ follows the following relationship:

$$
J \sim t\left(\frac{V}{t^{2}}\right)^{m}
$$

where $V, t$, and $m$ are the applied voltage, the sample thickness, and a fitting parameter, respectively [9]. Due to the presence of traps and interface states, $m$ is equal to about 3.8 for our device. This SCLC does not include the electrons charging to and 
from the $\mathrm{Cu}$ axle, which initiates the rotation movement along with the redox reaction. As the voltage is further increased, the current increases, possibly due to the other conduction paths such as the transport through misaligned rotator ligand molecules. These conduction paths have different voltage dependences. Better process control and proper characterization methods can decrease the misalignment probability to improve the performance stability. With smaller device sizes, the effect of the misaligned ligand becomes smaller (by the confinement of the structure). Therefore, the on/off ratio will be larger within the tunneling-transport regime.

Density functional theory calculations combined with the results of the absorption spectrum were obtained to estimate the energy levels of molecules, which give out about 3.4 and $2.7 \mathrm{eV}$ bandgaps for $\mathrm{Cu}(\mathrm{II})$ and $\mathrm{Cu}(\mathrm{I})$, respectively. Given that the computations were performed on the model compound $\mathrm{Cu}$ (2,9-dimethyl-1, 10-phenanthroline $)_{2}$, we did not include the effects of the Si substrate or of the gold electrode. The model used here is based on the gas phase instead of solid form as a reference to study our hypothesis of the transport mechanism. We believe that the computational results are relevant only as guidance to the experiment [10]. At the bias of $\sim 2.4 \mathrm{~V}$, the electron overcomes the high energy barrier of the $\mathrm{Cu}$ (II) and is transferred from the substrate into the empty states of $\mathrm{Cu}(\mathrm{II})$. This reduction process causes the conformational change as well as the increase of energy of the states. Thus, upon reduction and the associated rotational motion of $\mathrm{Cu}$ (II) to $\mathrm{Cu}(\mathrm{I})$, the bandgap reduces from about $3.4 \mathrm{eV}$ of $\mathrm{Cu}(\mathrm{II})$ to $2.7 \mathrm{eV}$ of $\mathrm{Cu}(\mathrm{I})$, causing the abrupt switching. This increases the transport probability, hence, results in the larger conduction current. The device operates in State 2 (LRS) until the application of a threshold negative bias causes the oxidation of $\mathrm{Cu}(\mathrm{I})$ to $\mathrm{Cu}(\mathrm{II})$. The latter process increases the bandgap by discharging one electron. Current flow then decreases, resulting in switching back to State 1.

To further study the electron-transport process during device operation, temperature-dependent $I-V$ measurements of the device were performed. Switching effects were no longer observed at temperatures below approximately $244 \mathrm{~K}$. Plotting the ON-state current over this temperature range revealed an exponential relationship at $T>244 \mathrm{~K}$, as shown in Fig. 4. Based on the well-known Arrhenius equation

$$
I \propto I_{0} \exp \left(\frac{-E_{a}}{k_{B} T}\right)
$$

where $E_{a}$ is the activation energy and $k_{B}$ is the Boltzmann's constant, curve fitting of the data acquired at $T>244 \mathrm{~K}$ yielded a rotational activation energy of approximately $0.3 \mathrm{eV}$, a value consistent with the theoretical quenching energy of rotation in the solution [11]. Such agreement between experiment and theory further supports the proposal of attributing the observed switching effect in the device to rotational motion within the molecular layer. It is recognized that the activation energy of $0.3 \mathrm{eV}$ is interpreted as the rotation energy from the fact that upon redox, there is a $90^{\circ}$ rotation between the two states. However, there are other effects, including the works related to sticking $\mathrm{Cu}(\mathrm{I})$ or $\mathrm{Cu}(\mathrm{II})$, which may affect the energy of the conformational change. For the OFF-state current, the observation of a weak temperature dependence indicates electron tunneling between the two device electrodes.

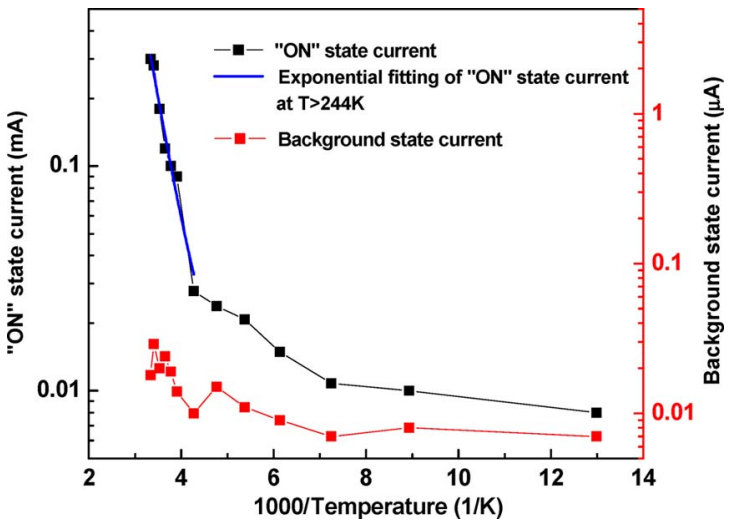

Fig. 4. Arrhenius plot of the ON-state current at $1 \mathrm{~V}$. The activation energy of rotation was extracted to be $0.3 \mathrm{eV}$ by exponentially fitting the curve of the measurement data higher than $244 \mathrm{~K}$. The weak temperature dependence of the background current with the device off at very small voltage implies tunneling transport of electrons.

\section{CONCLUSION}

In summary, a novel electrically driven molecular-rotor device has been fabricated and demonstrated for memory applications. The device shows an on/off ratio on the order of $10^{4}$, a read window of $2.5 \mathrm{~V}$, and retention performance of better than $10^{4} \mathrm{~s}$. The combined experimental data analysis as well as the observed temperature dependence of the switching behavior suggests the source of the observed switching effects to be redox-induced ligand rotation around the copper metal center. This organic device holds a potential for nonvolatile high-density memory applications due to its scalability and lower cost.

\section{REFERENCES}

[1] A. Aviram and M. A. Ratner, "Molecular rectifiers," Chem. Phys. Lett., vol. 29, no. 2, pp. 277-283, Nov. 1974.

[2] M. A. Reed, D. W. Bennett, J. Chen, D. S. Grubisha, L. Jones, II, A. M. Rawlett, J. M. Tour, and C. Zhou, "Prospects for molecular-scale devices," in IEDM Tech. Dig., 1999, pp. 227-230.

[3] R. Sezi, A. Walter, R. Engl, A. Maltenberger, J. Schumann, M. Kund, and C. Dehm, "Organic materials for high-density non-volatile memory applications," in IEDM Tech. Dig., 2003, pp. 259-262.

[4] Y. Song, Q. D. Ling, C. Zhu, E. T. Kang, D. S. H. Chan, Y. H. Wang, and D. L. Kwong, "Memory performance of a thin-film device based on a conjugated copolymer containing fluorine and chelated europium complex," IEEE Electron Device Lett., vol. 27, no. 3, pp. 154-156, Mar. 2006.

[5] H. T. Lin, Z. Pei, and Y. J. Chan, "A new nonvolatile bistable polymernanoparticle memory device," IEEE Electron Device Lett., vol. 28, no. 11, pp. 569-571, Nov. 2007.

[6] M. A. Reed, J. Chen, A. M. Rawlett, D. W. Price, and J. M. Tour, "Molecular random access memory cell," Appl. Phys. Lett., vol. 78, no. 23, pp. 3735-3737, Jun. 2001.

[7] S. Kabehie, A. Z. Stieg, M. Xue, M. Liong, K. L. Wang, and J. I. Zink, "Surface immobilized heteroleptic copper compounds as state variables," J. Phys. Chem. Lett, vol. 1, no. 3, pp. 589-593, Feb. 2010.

[8] W. F. Brinkman, R. C. Dynes, and J. M. Rowell, "Tunneling conductance of asymmetrical barriers," J. Appl. Phys., vol. 41, no. 5, pp. 1915-1921, Apr. 1970.

[9] M. C. Petty, Molecular Electronics: From Principles to Practice. Chichester, U. K.: Wiley, 2007

[10] S. Datta, W. Tian, S. Hong, R. Reifenberger, J. I. Henderson, and C. P. Kubiak, "Current-voltage characteristics of self-assembled monolayers by scanning tunneling microscopy," Phys. Rev. Lett., vol. 79, no. 13, pp. 2530-2533, Sep. 1997.

[11] D. V. Scaltrito, D. W. Thompson, J. A. Ocallaghan, and G. J. Meyer, "MLCT excited states of cuprous bis-phenanthroline coordination compounds," Coordination Chem. Rev., vol. 208, no. 1, pp. 243-266, Oct. 2000. 